\title{
UML Entity
}

National Cancer Institute

\section{Source}

National Cancer Institute. UML Entity. NCI Thesaurus. Code C41000.

caBIO domain objects represent entities found in biomedical research. The objects simulate the behavior of components such as genes, chromosomes, sequences, libraries, clones, and ontologies. The caBIO objects include the behavior (operations) and the relationships that the actual bioinformatics objects have, for example, a gene can retrieve its EST 's, SNP's, or clones. (from National Cancer Institute Center for Bioinformatics) 